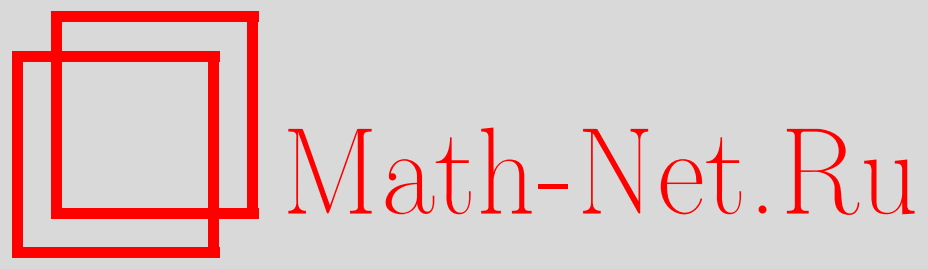

С. В. Кисляков, Д. В. Максимов, Д. М. Столяров, Пространства гладких функций, порожденные неоднородными дифференциальными выражениями, Функи. анализ и его прил., 2013, том 47, выпуск 2, 89-92

DOI: https://doi.org/10.4213/faa3114

Использование Общероссийского математического портала MathNet.Ru подразумевает, что вы прочитали и согласны с пользовательским соглашением

http://www . mathnet.ru/rus/agreement

Параметры загрузки:

IP : 35.173 .137 .237

26 апреля 2023 г., $07: 23: 22$

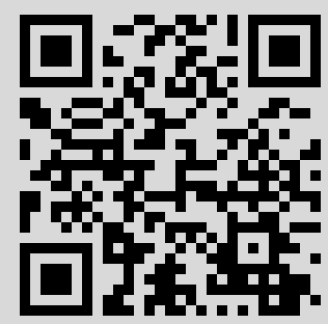


[6] Y. Derriennic, in: Conference on Random Walks (Kleebach, 1979), Astérisque, vol. 74, Soc. Math. France, Paris, 1980, 183-201, 4. [7] A. Erschler, in: Random walks, boundaries and spectra. Proceedings of the workshop on boundaries, Graz, Austria, June 29-July 3, 2009 and the Alp-workshop, Sankt Kathrein, Austria, July 4-5, 2009, Progress in Probability, vol. 64 (eds. Lenz, Daniel et al.), Birkhäuser, Basel, 2011, 55-64. [8] H. Furstenberg, Trans. Amer. Math. Soc., 108 (1963), 377-428. [9] Sur les groupes hyperboliques d'après Mikhael Gromov, Papers from the Swiss Seminar on Hyperbolic Groups held in Bern, 1988, Progress in Mathematics, vol. 83 (eds. É. Ghys, P. de la Harpe), Birkhäuser, Boston, MA, 1990. [10] M. Gromov, Hyperbolic groups, in: Essays in group theory, Math. Sci. Res. Inst. Publ., vol. 8, Springer-Verlag, New York, 1987, 75-263. [11] V. A. Kaimanovich, C. R. Acad. Sci. Paris Sér. I Math., 318:1 (1994), 59-64. [12] V. A. Kaimanovich, Ann. of Math. (2), 152:3 (2000), 659-692. [13] V. A. Kaimanovich, A. M. Vershik, Ann. Probab., 11:3 (1983), 457-490. [14] F. Ledrappier, in: Topics in probability and Lie groups: boundary theory, CRM Proc. Lecture Notes, vol. 28, Amer. Math. Soc., Providence, RI, 2001, 117152. [15] F. Ledrappier, http://arxiv.org/abs/1110.3156. [16] F. Ledrappier, Groups Geom. Dyn., 6:2 (2012), 317-333. [17] P. Mathieu, http://arxiv.org/abs/1209.2020. [18] J. Mairesse, F. Mathéus, J. Lond. Math. Soc. (2), 75:1 (2007), 47-66.

Department of Mathematics and Statistics,

University of Ottawa

Поступило в редакцию

e-mail: vkaimano@uottawa.ca

2 июля 2012 г.

C.N.R.S., Département de Mathématiques,

Université Paris Sud, Orsay, France

e-mail: anna.erschler@math.u-psud.fr

УДК 517.982

\section{Пространства гладких функций, порожденные неоднородными дифференциальными выражениями*}

\section{(c) 2013. С. В. Кисляков, Д. В. Максимов, Д. М. Столяров}

Пространства непрерывно дифференцируемых функций на многообразиях размерности по крайней мере 2 (здесь мы ограничимся торами) в невырожденных случаях не изоморфны пространству $C(K)$ ни для какого компакта $K$ (по теореме Милютина то же самое можно выразить, сказав «не изоморфны пространству $C[0,1] »)$. Смысл этого высказывания уточнялся постепенно, история вопроса была подробно изложена в [3]. Мы обратимся сразу к общей ситуации, описанной в [3]. Пусть $T=\left(T_{1}, \ldots, T_{l}\right)$ - набор дифференциальных операторов с постоянными коэффициентами на торе $\mathbb{T}^{n}$. (Частная производная $\partial_{k}$ на торе - это оператор $g \mapsto \frac{\partial}{\partial t} g\left(e^{2 \pi i t}\right), t \in \mathbb{R}$, применяемый по $k$-й переменной.) На множестве тригонометрических полиномов от $n$ переменных введем полунорму $\|f\|_{T}=\max _{1 \leqslant j \leqslant l}\left\|T_{j} f\right\|_{C\left(\mathbb{T}^{n}\right)}$. После факторизации по ядру и пополнения получаем пространство $C^{T}\left(\mathbb{T}^{n}\right)$.

Если набор $T$ состоит из всех дифференциальных мономов порядка не выше $s$, возникает обычное пространство $C^{(s)}\left(\mathbb{T}^{n}\right)$, которое действительно не вкладывается дополняемо в $C[0,1]$ при $n \geqslant 2$ и $s \geqslant 1$, см., например, [2]. (Умест-

*Работа выполнена при поддержке Лаборатории Чебышева СПБГУ (грант Правительства РФ, дог. 11.G34.31.0026) и РФФИ (грант 11-01-00526). 
но напомнить, что старая задача об изоморфной классификации пространств $C^{(s)}\left(\mathbb{T}^{n}\right)$ до сих пор не решена, неизвестно даже, изоморфны ли пространства $C^{(1)}\left(\mathbb{T}^{2}\right)$ и $C^{(1)}\left(\mathbb{T}^{3}\right)$.)

Если $T$ - произвольный конечный набор дифференциальных мономов, мы имеем дело с анизотропным пространством гладких функций. Тогда по-прежнему $C^{T}\left(\mathbb{T}^{n}\right)$ не вкладывается дополняемо в $C[0,1]$, если среди мономов есть два, у которых мультииндексы максимальны и несравнимы относительно покоординатного порядка (см. [6], [7]).

Пусть операторы набора $T$ не обязательно сводятся к мономам и все имеют порядок не выше $s>0$. Отбросим в каждом операторе $T_{j}$ все дифференциальные мономы порядка строго меньше $s$, останется его главная часть. В [3], [4] было показано, что если среди главных частей есть хотя бы две непропорииональные, то пространство $C^{T}\left(\mathbb{T}^{n}\right)$ не вкладывается дополняемо в $C(K)$.

Заметим, что если все главные части пропорциональны одной из них, изоморфизма все равно может не быть - например, его нет для набора $\left(\partial_{1}, \partial_{2}^{2}\right)$ в случае двумерного тора (см. цитированное утверждение об анизотропных пространствах). Следующая теорема обобщает результаты работ [7], [6] и [3], [4].

Пусть $\Lambda$ - гиперплоскость с уравнением $\sum_{k=1}^{n} x_{k} / a_{k}=1, a_{k}>0$. Предположим, что неравенство $\sum_{k=1}^{n} \alpha_{k} / a_{k} \leqslant 1$ выполняется для каждого дифференциального монома $\partial_{1}^{\alpha_{1}} \cdots \partial_{n}^{\alpha_{n}}$, встречающегося хотя бы в одном операторе $T_{j}$, $j=1, \ldots, l$. В каждом операторе $T_{j}$ выделим слагаемые, для которых неравенство обращается в равенство. Их сумма называется $\Lambda$-главной частью оператоpa $T_{j}$.

Теорема 1. Если хотя бъ для одной гиперплоскости $\Lambda$ среди всех $\Lambda$-главнъх частей операторов из набора $T$ найдутся две непропорииональные, то пространство $C^{T}\left(\mathbb{T}^{n}\right)$ не вкладьвается дополняемо в $C[0,1]$.

Во всех цитированных статьях доказательство отсутствия изоморфизма основывалось на соболевских вложениях с предельным показателем (в размерности 2). Так происходит и в нашем случае, однако соответствующая теорема вложения нестандартна. Введем соболевское пространство с дробной гладкостью на двумерном торе $W_{2}^{\alpha, \beta}\left(\mathbb{T}^{2}\right)=\left\{f:\left(1+m^{2}\right)^{\alpha / 2}\left(1+n^{2}\right)^{\beta / 2} \hat{f}(m, n) \in l^{2}\left(\mathbb{Z}^{2}\right)\right\}$. Распределение $f$ на торе $\mathbb{T}^{2}$ назовем правильным, если $\hat{f}(s, t)=0$, как только $s=0$ или $t=0, s, t \in \mathbb{Z}$.

Теорема 2. Пусть $k$ и $l$ - натуральные числа, одно из которьх нечетно, а правильные распределения $\varphi_{1}, \ldots, \varphi_{N}$ на двумерном торе удовлетворяют системе уравнений

$$
-\partial_{1}^{k} \varphi_{1}=\mu_{0}, \quad \partial_{2}^{l} \varphi_{j}-\partial_{1}^{k} \varphi_{j+1}=\mu_{j}, \quad j=1, \ldots, N-1, \quad \partial_{2}^{l} \varphi_{N}=\mu_{N},
$$

где $\mu_{0}, \ldots, \mu_{N}-$ мерьь. Тогда

$$
\sum_{j=1}^{N}\left\|\varphi_{j}\right\|_{W_{2}^{\alpha, \beta}\left(\mathbb{T}^{2}\right)} \leqslant C \sum_{j=0}^{N}\left\|\mu_{j}\right\|
$$

при $\alpha=(k-1) / 2, \beta=(l-1) / 2$. Под нормой меры понимается ее полная вариачия.

Аналогичное утверждение верно и для плоскости.

Теорема 3. Пусть $\varphi_{j}, j=1, \ldots, N$, - распределения с компактным носителем на плоскости $\mathbb{R}^{2}$. Предположим, что они удовлетворяют системе (1), 
где $\mu_{0}, \ldots, \mu_{N}$ - конечные меры с компактным носителем. Тогда верен аналог оценки (2), если соболевское пространство на плоскости понимать так: $W_{2}^{\alpha, \beta}\left(\mathbb{R}^{2}\right)=\left\{f:|\xi|^{\alpha}|\eta|^{\beta} \hat{f}(\xi, \eta) \in L^{2}\left(\mathbb{R}^{2}\right)\right\}$.

Взятые вместе, теоремы 2 и 3 составляют второй основной результат работы. Они отличаются от классических теорем вложения тем, что условие быть мерой наложено на линейные комбинации производных разных порядков от различных функций, а не на производные одной и той же функции. Для случая $k=l=1$ обе теоремы были доказаны в работе [3]. При $k \neq l$ приходится действовать иным, более сложным образом. Если отвлечься от способа доказательства, то сами формулировки теорем 2 и 3 можно рассматривать как смесь теоремы вложения из [3] и хорошо известной теоремы вложения для анизотропных пространств Соболева, см. [8], [7].

Что произойдет, если условия теоремы 1 (для простоты считаем, что $n=2$, поскольку теорема 1 сводится к этому случаю) нарушаются? Для анизотропных пространств тогда имеет место изоморфизм пространству $C[0,1]$, см. [6], [7]. В общем случае ситуация не так проста (это отмечалось еще в [3]-[5]). Мы можем доказать, что при нарушении условий теоремы 1 изоморфизм пространству $C[0,1]$ имеет место при некотором дополнительном требовании «эллиптичности». Мы не приводим точной формулировки. Вместо этого займемся примерами.

Прежде всего, рассмотрим пространство, порожденное двумя операторами $T_{1}=\partial_{1}^{2}+2 \partial_{1} \partial_{2}+\partial_{2}^{2}$ и $T_{2}=a \partial_{1}+b \partial_{2}$, где $a$ и $b-$ комплексные числа. Henoсредственно теорема 1 ничего не сообщает о нем. Кроме того, первый оператор не «эллиптичен» (для однородного оператора - кстати, неважно, с обычной или смешанной однородностью - «эллиптичность» означает отсутствие вещественных нулей у характеристического полинома всюду, кроме координатных осей). Однако после замены переменных $\theta_{1}=t_{1}+t_{2}, \theta_{2}=t_{2}$ операторы $T_{1}$ и $T_{2}$ превращаются соответственно в $\left(\partial / \partial t_{2}\right)^{2}$ и $(a-b) \partial / \partial t_{1}+b \partial / \partial t_{2}$. Теперь, если $a \neq b$, применима теорема 1 о неизоморфизме (рассмотрим прямую, проходящую через точки $(1,0)$ и $(0,2))$. Если же $a=b$, то совсем легко усмотреть, что имеет место изоморфизм пространству $C\left(\mathbb{T}^{2}\right)$.

Следующий пример связан с теоремой Коэна об идемпотентах (см. монографию [1]). Ситуация обсуждалась в [5], мы не повторяем объяснений, а отметим только, что если набор $T$ состоит из единственного оператора $2 \pi i \partial_{1}-\partial_{2}^{2}$ (или, например, оператора $(2 \pi)^{2} \partial_{1}+\partial_{2}^{3}$ с вещественными коэффициентами), то пространство $C^{T}\left(\mathbb{T}^{2}\right)$ не вкладывается дополняемо в $C[0,1]$. Причина, впрочем, не имеет особого отношения к гладкой структуре.

Наконец, нам ничего не известно про случай набора из двух операторов (Id, $\left.\partial_{1}-\sqrt{2} \partial_{2}\right)$. Второй оператор не «эллиптичен» (у характеристического полинома есть вещественные нули вне координатных осей). Иначе говоря, не помогает ни несформулированная теорема об изоморфизме, ни теорема 1. Линейнорациональные замены переменных (как в первом примере) здесь тоже бесполезны.

\section{ЛитЕРАТУРА}

[1] C. C. Graham, O. C. McGehee, Essays in Commutative Harmonic Analysis, SpringerVerlag, Berlin, 1979. [2] С. В. Кисляков, Функц. анализ и его прил., 9:4 (1975), $22-$ 27. [3] С. В. Кисляков, Д. В. Максимов, Зап. научн. сем. ПОМИ, 327 (2005), $78-97$. [4] С. В. Кисляков, Д. В. Максимов, Препринт ПОМИ 6/2009. [5] Д. В. Максимов, 
Зап. научн. сем. ПОМИ, 333 (2006), 62-65. [6] С. В. Кисляков, Н. Г. Сидоренко, Сибирск. матем. ж., 29:3 (1988), 64-77. [7] A. Pełczyński, K. Senator, Studia Math., 84:2 (1986), 169-215. [8] В. А. Солонников, Зап. научн. сем. ЛОМИ, 27 (1972), 194-210.

С.-Петербургское отделение Математического института им. В. А. Стеклова

Поступило в редакцию e-mail: skis@pdmi.ras.ru

1 августа 2012 г.

С.-Петербургский Государственный

Политехнический Университет

e-mail: dimax239@bk.ru

Исследовательская лаборатория им. П. Л. Чебышева

СПБГУ, Санкт-Петербург

e-mail: dms239@mail.ru

УДК 517.98

\section{Ординарные полукаскады и их эргодические свойства}

(C) 2013. A. B. Романов

Введение. В этой статье эргодические свойства дискретной динамической системы $(\Omega, \varphi)$, порожденной непрерывным преобразованием метрического компакта $\Omega$, формулируются в терминах трех ассоциированных с $(\Omega, \varphi)$ алгебротопологических объектов. Речь идет об описанных в [1], [2] обволакивающей полугруппе Эллиса $E(\Omega, \varphi)$ и операторной обволакивающей полугруппе Кёлер $\Gamma(\Omega, \varphi)$, а также, о рассмотренной в предшествующей работе автора [3] и связанной с $\Gamma(\Omega, \varphi)$ операторной полугруппе $G(\Omega, \varphi)$. Помимо этого изучаются представляющие самостоятельный интерес зависимости между тремя данными полугруппами. Как показано в [3], топологические характеристики компактных в соответствующих топологиях полугрупп $E(\Omega, \varphi), \Gamma(\Omega, \varphi)$ и $G(\Omega, \varphi)$ тесно связаны с эргодическими свойствами полукаскада $(\Omega, \varphi)$. Оказывается, подобного рода связи проявляются особенно ярко для класса дискретных динамических систем, обладающих метризуемой обволакивающей полугруппой $E(\Omega, \varphi)$ и называемых здесь ординарными. Ряд результатов о полугруппах Эллиса подобных систем (причем не только дискретных) можно найти в обзоре [4]. Известно, что ординарными оказываются все слабо почти периодические динамические системы.

Здесь предложено (теорема 4) альтернативное определение ординарности полукаскада $(\Omega, \varphi)$, состоящее в требовании метризуемости индуцированной полугруппы $G(\Omega, \varphi)$. Рассматривается также первоначально введенный Кёлер [2] и изученный в работах [4]-[6] класс так называемых ручных динамических систем $(\Omega, \varphi)$. В разд. 2 представлена иерархическая сводка известных ранее и некоторых новых свойств полукаскада $(\Omega, \varphi)$, формулируемых большей частью в терминах полугрупп $E(\Omega, \varphi), \Gamma(\Omega, \varphi)$ и $G(\Omega, \varphi)$.

В то же время главные результаты заметки относятся к слабой* сходимости операторных эргодических средних для ординарных и ручных полукаскадов. Сама идея рассмотрения подобной сходимости в эргодической теории восходит по существу к классической работе Крылова и Боголюбова [7]. 